\title{
Assessment of Knowledge and Attitude of Expectant Mothers Regarding Effect of Their Oral Health and Its Influence on the Infant Oral Health
}

\author{
Kirti Pattanshetti ${ }^{1}$, Harshita P Kothari ${ }^{2}$, Jayesh Tiwari ${ }^{3}$, Sachin Malagi ${ }^{4}$, Spoorti Pattanashetty ${ }^{5}$, Kiran Hinge ${ }^{6}$
}

\begin{abstract}
Introduction: Good oral health during pregnancy and infancy is important for the overall health and well-being of mother and child, respectively. Aim: To assess the knowledge and attitude of expectant mothers regarding the effect of their oral health and its influence on infant oral health. Materials and methods: A cross-sectional study was conducted among expectant mothers. They were randomly selected from those reporting to the Department of Gynecology in Government and Private Hospitals in Durg, Chhattisgarh. A total of 124 closed-ended, self-administered questionnaire were filled by participants, which had 21 items addressing the knowledge and attitude of expectant mothers regarding their oral health and oral hygiene practices; the transmission of bacteria from mother to child; cariogenic food consumption; effect of periodontal disease on infant's teeth and safe trimester for dental treatment. Data were tabulated and subjected to statistical analysis.

Results: Significant differences were found in expectant mothers' knowledge and attitude based on their age $(p=0.02,0.07)$, education level $(p=0.001,0.95)$, trimester $(p=0.83,0.43)$, and number of pregnancies $(p=0.05,0.10)$, respectively.

Conclusion: The knowledge related to the oral health of pregnant women during pregnancy was found to be low and needs to be improved. Clinical significance: Execution of educational programs along with other healthcare professionals to motivate expectant mothers.

Keywords: Infant oral health, Maternal oral health, Pregnancy, Trimester.

International Journal of Clinical Pediatric Dentistry (2020): 10.5005/jp-journals-10005-1817
\end{abstract}

\section{INTRODUCTION}

The mother-child relationship serves as a salient foundation from which both men and women develop their secure base and thus their general emotional well-being. ${ }^{1}$ According to the United Nations Children's Fund (UNICEF), at least $20 \%$ of the disease burden in children under 5 is related to problems in maternal health and malnutrition, as well as the quality of care at delivery and during the newborn period. ${ }^{2}$

The American Academy of Pediatric Dentistry (AAPD) recognizes that perinatal and infant oral health are the foundations upon which preventive education and dental care must be built to enhance the opportunity for a lifetime free from preventable oral disease. ${ }^{3}$ Maternal oral health has a significant impact on her child's oral health. Mothers are often referred to as the "key figure" in their child's general and dental health as they are usually the primary caregivers. ${ }^{4}$

Pregnancy is a remarkable period in a woman's life and is described by complex physiological, emotional, and hormonal changes. ${ }^{5}$ According to a systematic review conducted by Gambhir et al., pregnant women's knowledge and awareness regarding oral health was poor, in which most of the women were unaware of the potential consequences of neglecting oral hygiene during pregnancy. ${ }^{6}$ Most of the studies were done to assess the knowledge, attitude, and practice of mothers regarding the oral health of preschool children. ${ }^{7-10}$

The knowledge and attitude toward oral health among pregnant women can be a strong predictor of not only her oral health but also her future infant's oral health. Growing evidence shows that general health and good oral health of mothers right from pregnancy could be the key to establishing good infant oral

\begin{abstract}
${ }^{1-3}$ Department of Pedodontics and Preventive Dentistry, Maitri College of Dentistry and Research Centre, Chhattisgarh, India

${ }^{4}$ Department of Periodontics, Maitri College of Dentistry and Research Centre, Chhattisgarh, India

${ }^{5}$ Department of Conservative Dentistry and Endodontics, Maitri College of Dentistry and Research Centre, Chhattisgarh, India

${ }^{6}$ All India Institute of Medical Science, Ansari Nagar, New Delhi, India

Corresponding Author: Kirti Pattanshetti, Department of Pedodontics and Preventive Dentistry, Maitri College of Dentistry and Research Centre, Chhattisgarh, India, Phone: +91 7987863623, e-mail: drkirthipattanshettimalgi@gmail.com

How to cite this article: Pattanshetti K, Kothari HP, Tiwari J, et al. Assessment of Knowledge and Attitude of Expectant Mothers Regarding Effect of Their Oral Health and Its Influence on the Infant Oral Health. Int J Clin Pediatr Dent 2020;13(5):471-475.

Source of support: Nil

Conflict of interest: None
\end{abstract}

health. ${ }^{11}$ Fewer studies have been reported on expectant mothers' knowledge, attitude, and practice to evaluate the effect of maternal oral health influence on a child's oral health. Thus, this study was undertaken with the purpose to assess the knowledge and attitude of expectant mothers regarding the effect of their oral health and its influence on infant oral health.

\section{Materials and Methods}

A cross-sectional study was conducted among randomly selected expectant mothers reporting to the Department of Gynecology in Government and Private Hospital in Durg, Chhattisgarh, in July 
2018. The study was conducted upon obtaining approval from the Institutional Review Board and Ethical Committee of Maitri College of Dentistry and Research Centre, Anjora, Durg. The study was briefed and consent was obtained from each of the participants before the study. Expectant mothers in the first, second, and third trimester were enrolled for the study and those not willing to participate were excluded.

The questionnaire was prepared in English, Hindi, and in the local language. A total of 124 closed-ended, self-administered questionnaires were filled by participants, which were divided into two parts.

- Part A-Demographic details of participants like age, education, trimester, and number of pregnancies.

- Part B-21 items addressing the knowledge and attitude of expectant mothers regarding the effect of their oral health on infants' oral health.

The filled questionnaire forms were collected from the respondents and were coded. All closed-ended questions were coded with numerical against responses. The data were entered into a Microsoft Excel sheet. This sheet was then analyzed using Statistical Packages for Social Sciences (SPSS), version 20.0 software. Results were expressed as the number and percentage of respondents for each question. Based on the responses received through questionnaires, the data obtained were analyzed using statistical data. The total number and percentage were calculated and then analysis was done using the Chi-square test and $t$-test. The Chi-square test is used to determine a significant difference between the expected frequencies and the observed frequencies in one or more categories and $t$-test was used to compare the mean scores. For the test, a $p<0.05$ is considered as statistically significant.

\section{Results}

A total of 124 expectant mothers participated in the questionnaire survey. The demographic profile, such as, age, educational qualification, trimester, and number of pregnancies, is shown in Table 1. Table 2 shows a comparison of mean scores of knowledge and attitude according to the age groups. The mean score of knowledge was more in the age group of $26-30$ years $(p=0.02)$ and those in $31-35$ years $(p=0.07)$ of age group had a positive attitude. Table 3 shows a comparison of mean scores of knowledge and attitude according to the educational qualification. The mean score of knowledge was more in postgraduates $(p=0.001)$. The attitude scores were comparable between all educational qualification groups $(p=0.95)$. Table 4 shows a comparison of mean scores of knowledge and attitude according to the trimester. The mean score of knowledge was more in the first trimester $(p=0.83)$. The attitude scores were comparable between all trimesters $(p=0.43)$. Table 5 shows a comparison of mean scores of knowledge and attitude according to the number of pregnancies. The mean score of knowledge was more in mothers with more than one pregnancy $(p=0.05)$, whereas there was a non-significant $(p=0.10)$ difference in attitude when the number of pregnancies was considered.

The overall score showed that the majority of the expectant mothers had poor knowledge and attitude regarding the effect of their oral health and its influence on infant oral health.

\section{Discussion}

Questions in the survey were regarding knowledge of expectant mothers' oral health and oral hygiene practices; the transmission
Table 1: Demographic details of the study population in terms of frequency and percentage

\begin{tabular}{llcc}
\hline Variables & & Frequency & Percentage \\
\hline Age & $20-25$ & 43 & 34.7 \\
& $26-30$ & 68 & 54.8 \\
& $31-35$ & 13 & 10.5 \\
Qualification & Total & 124 & 100 \\
& Below 7 standard & 16 & 12.9 \\
& 8th to pre-degree & 44 & 35.5 \\
& Graduate & 38 & 30.6 \\
Pregnancy period & 26 & 21.0 \\
& Postgraduate & 124 & 100 \\
& Total & 28 & 22.6 \\
& First trimester & 53 & 42.7 \\
& Second trimester & 43 & 34.7 \\
& Third trimester & 124 & 100 \\
& Total & 53 & 42.7 \\
No. of pregnancies & First & 71 & 57.3 \\
& More than one & 124 & 100.0 \\
\hline
\end{tabular}

Table 2: Comparison of mean scores of knowledge and attitude according to age groups

\begin{tabular}{lllll}
\hline & $\begin{array}{l}\text { Age (in } \\
\text { years) }\end{array}$ & $\begin{array}{l}\text { Frequency } \\
(n)\end{array}$ & Mean $\pm S D$ & pvalue \\
\hline Knowledge & $20-25$ & 43 & $1.0735 \pm 0.39836$ & 0.02 \\
& $26-30$ & 68 & $1.3721 \pm 0.53556$ & \\
Attitude & $31-35$ & 13 & $0.9231 \pm 0.49355$ & \\
& $20-25$ & 43 & $0.8372 \pm 0.37354$ & 0.07 \\
& $26-30$ & 68 & $0.6912 \pm 0.46544$ & \\
& $31-35$ & 13 & $0.9231 \pm 0.27735$ & \\
\hline
\end{tabular}

of bacteria from mother to child; cariogenic food consumption; effect of periodontal disease on infant's teeth and safe trimester for dental treatment.

Mother's oral hygiene, especially during pregnancy, plays an important role as it can have a direct influence on her infant's oral health. ${ }^{11}$ Optimal maternal oral health is a critical component in the overall health of the mother as well as in the success of a pregnancy and health of the child. ${ }^{12,13}$ Out of 124 participants, 57 (45.9\%) expectant mothers belonging to $26-30$ years of age group were aware regarding good oral health during pregnancy.

In the present study, $49.2 \%$ of participants agreed that the higher the caries index in mothers more is the chance of tooth decay in infants. This finding was under the study conducted by Chacko wherein $25.3 \%$ were found to have agreed about this relationship. ${ }^{14}$ $74.2 \%$ of expectant mothers agreed on the point that good oral hygiene practices during pregnancy will prevent oral health problems in infants. The results obtained from the present study revealed that $63.7 \%$ of participants believed that cariogenic food consumption during pregnancy can cause dental caries in children.

The American Dental Association (ADA), the American Congress of Obstetricians and Gynecologists, and the American Academy of Pediatrics all encourage women to get dental care while pregnant. ${ }^{15}$ Hormonal changes during pregnancy increase the risk of gingivitis, a milder form of and precursor to more severe periodontal disease. ${ }^{16}$ The American Academy of Periodontology (AAP) reports that half of the women experience gingivitis during pregnancy and 
Assessment of Knowledge and Attitude of Expectant Mothers

Table 3: Comparison of mean scores of knowledge and attitude according to educational qualification

\begin{tabular}{lllll}
\hline & Educational qualification & Frequency $(n)$ & Mean \pm SD & p value \\
\hline Knowledge & Below 7th standard & 16 & $1.0000 \pm 0.00000$ & $0.001(\mathrm{HS})$ \\
& 8th to pre-degree & 44 & $1.2273 \pm 0.56501$ & \\
& Graduate & 38 & $1.1053 \pm 0.38831$ & \\
Attitude & Postgraduate & 26 & $1.3750 \pm 0.71880$ & \\
& Below 7th standard & 16 & $0.7500 \pm 0.44721$ & 0.95 \\
& 8th to pre-degree & 44 & $0.7727 \pm 0.42392$ & \\
& Graduate & 38 & $0.7895 \pm 0.41315$ & \\
& Postgraduate & 26 & $0.7308 \pm 0.45234$ & \\
\hline
\end{tabular}

Table 4: Comparison of mean scores of knowledge and attitude according to trimester

\begin{tabular}{lllll}
\hline & Trimester & $N$ & Mean \pm SD & p value \\
\hline Knowledge & First trimester & 28 & $1.2143 \pm 0.49868$ & 0.83 \\
& Second trimester & 53 & $1.1887 \pm 0.48265$ & \\
& Third trimester & 43 & $1.0930 \pm 0.47879$ & \\
Attitude & First trimester & 28 & $0.8571 \pm 0.35635$ & 0.43 \\
& Second trimester & 53 & $0.7358 \pm 0.44510$ & \\
& Third trimester & 43 & $0.7442 \pm 0.44148$ & \\
\hline
\end{tabular}

Table 5: Comparison of mean scores of knowledge and attitude according to number of pregnancies

\begin{tabular}{lllll}
\hline & No. of pregnancies & Frequency $(n)$ & Mean \pm SD & p value \\
\hline Knowledge & First & 53 & $1.0704 \pm 0.45744$ & $0.05(S)$ \\
& More than one & 71 & $1.2830 \pm 0.49526$ & \\
Attitude & First & 53 & $0.8302 \pm 0.37906$ & 0.10 \\
& More than one & 71 & $0.7183 \pm 0.45302$ & \\
\hline
\end{tabular}

recommends that women should visit the dentist for a periodontal evaluation before pregnancy and that they maintain oral hygiene during pregnancy. ${ }^{17}$ In this study, $56.5 \%$ of expectant mothers knew the importance of dental visits during pregnancy. Only $2.4 \%$ of them have consulted the dentist during the second trimester, which was in agreement with the survey conducted by Thomas et al. in which they reported only $6.1 \%$ of participants had consulted the dentist. The cause for lack of positive attitude to visit the dentist could be due to more concern toward the general health than oral health and anxiety and stress while undergoing dental treatment.

Dental care during pregnancy is safe, and there are appropriate guidelines for the treatment of pregnant patients. ${ }^{18}$ The American Dental Association acknowledges that preventive, diagnostic, and restorative dental treatment to promote health and eliminate the disease is safe throughout pregnancy and is effective in improving and maintaining the oral health of the mother and her child. ${ }^{19}$ Some elective and emergent dentoalveolar procedures are more safely accomplished during the second trimester. Although there is no risk to the fetus during the third trimester, the pregnant mother may experience an increased level of discomfort. Short dental appointments should be scheduled with the appropriate positioning of expectant mothers on the dental chair. ${ }^{18}$ In this study only 8 out of 124 participants were aware of the safe period for undergoing dental treatment during pregnancy.

An adequate diet during gestation is important for the optimal oral development of the fetus. Nutrients of particular importance include folate (folic acid), calcium, magnesium, zinc, and vitamins $\mathrm{K}, \mathrm{C}, \mathrm{B}-6$, and B-12. Maternal levels of vitamin D during pregnancy may affect the developing primary dentition, with lower levels altering enamel integrity and increasing the risk for early childhood caries. ${ }^{20}$ The results of this study showed that $76.6 \%$ of expectant mothers agreed that an unhealthy diet during pregnancy can affect a child's primary teeth.

Prenatal maternal cigarette smoking (PMCS) has been associated with complications including ectopic pregnancy, spontaneous abortion, preterm birth, low birth weight, birth defects, and sudden infant death syndrome. ${ }^{21}$ Increasing evidence shows that maternal tobacco use is associated with intellectual disability and birth defects, such as, oral clefts. ${ }^{20}$ Children exposed to alcohol in utero manifest growth deficiency, central nervous system manifestations, and congenital malformations, the most prominent being craniofacial malformations. ${ }^{22}$ Substance abuse in pregnancy is associated with significant maternal and fetal morbidity. Prenatal use of illicit drugs increases the rate of spontaneous abortion, abruptio placentae, premature labor and delivery, intrauterine growth retardation, sudden infant death syndrome, neurobehavioral deficiencies, as well as long-term developmental abnormalities. ${ }^{23} 74.2 \%$ of expectant mothers agreed that tobacco smoking/drugs increase the risk of oral health problems in infants/children.

Mothers are generally responsible for the implementation of oral health behaviors in their children. If mothers exhibit deficient habits, it is plausible that their children will also exhibit unfavorable oral health habits. ${ }^{24}$ The results of this study demonstrated that $50.8 \%$ of the expectant mothers brushed twice daily; however, this figure was lower when compared to the studies conducted by Christensen et al., ${ }^{25}$ Hullah, ${ }^{26}$ and Assery et al. ${ }^{27}$ who reported $96,73.7$, and $77 \%$, respectively. However, results were similar to 
surveys conducted by Ganesh et al. ${ }^{28}$ and Thomas et al. ${ }^{11}$ reported that 66 and $64 \%$ of expectant mothers practiced brushing twice daily, respectively.

Acquisition of Streptococcus mutans can occur through both vertical and horizontal transmission. ${ }^{29} \mathrm{~A}$ systematic review and meta-analysis concluded that there was an association between S. mutans mothers and their respective children through vertical transmission. ${ }^{30}$ The present study showed that only $7.3 \%$ of expectant mothers were aware of the transmission of bacteria causing dental caries from mother to child by kissing or sharing utensils. This response was not under the survey conducted by Thomas et al. (22.2\%) and Shenoy et al. which reported that $35.6 \%$ of participants knew about the transmission of bacteria from mother to child. ${ }^{11,14}$

Maternal periodontal disease is a highly prevalent condition that has been associated with adverse pregnancy outcomes, including preterm delivery, preeclampsia, and low birth weight. ${ }^{31}$ Periodontal disease can initiate the spread of bacteria and host mediators to the developing fetus through the blood to induce pregnancy-related complications. ${ }^{32}$ These bacteria can travel to the uterus, triggering the production of chemicals called prostaglandins, which are suspected to induce premature labor. ${ }^{33}$ Studies have demonstrated an association between adverse pregnancy outcomes and developmental defects of enamel (DDE) in children. ${ }^{34,35}$ Most of the expectant mothers did not acknowledge that periodontal gum diseases during pregnancy contribute toward the low birth weight babies and premature births which may lead to DDE in infants. In this study, only $12.1 \%$ of participants knew this.

The results of the present study showed that expectant mothers in the age group 26-30 years were more aware regarding the influence of their oral health on infant oral health (Table 2, $p=$ 0.02 ) and those in $31-35$ years (Table $2, p=0.07$ ) of age group had a positive attitude.

In the present study, expectant mothers with higher educational qualification had better knowledge (Table 3, $p=$ 0.001 ) which was similar to studies conducted by Thomas et al., ${ }^{11}$ Sufia, $^{36}$ and Mascarenhas. ${ }^{37}$ This can be attributed to the fact that improved level of education of mothers makes them able to assess the appropriate source of information and understand that information more completely.

The results of this study revealed that expectant mothers in their first trimester had better knowledge and a positive attitude compared to mothers in the second and third trimester with no significant difference (Table $4, p=0.83,0.43$ ).

The results of this study showed that mothers with more than one pregnancy had better knowledge (Table 5, $p=0.05$ ) compared to mothers with first pregnancy. This might be due to an increase in the level of understanding and experience with an increase in the number of children. Also, mothers with a child are better able to understand the relationship between a child's diet and oral disease.

In this study, $85.5 \%$ of expectant mothers showed a positive attitude for attending educational sessions on infant oral health. Mothers are responsible for their child's lifestyle, behavior, and habits and represent the primary information about oral health. Hence, pre- and postnatal counseling of mothers about oral health will help in increasing the oral health awareness of their children. ${ }^{38}$

\section{Limitations}

The limitation of the present study is convenience sampling and small sample size which was limited to Durg city, hence findings may not be generalized to other cities in Chhattisgarh and India.

\section{Conclusion}

The knowledge related to the oral health of pregnant women during pregnancy was found to be low and needs to be improved. Execution of educational programs to motivate expectant mothers and bring out awareness about the importance of oral health, healthy diet, and their implications must be emphasized.

\section{References}

1. Mallers MH, Charles ST, Neupert SD, et al. Perceptions of childhood relationships with mother and father: daily emotional and stressor experiences in adulthood. Dev Psychol 2010;46(6):1651-1661. DOI: 10.1037/a0021020.

2. Clemente V, Bueno C, Almeida P. Maternal health: the importance of caring for mothers is a crucial factor in the health of children. Available from www.evidencias.com.

3. Perinatal and infant oral health care. Am Acaed Pediat Dentis 2016;40(6):228-232.

4. Shearer DM. Does maternal oral health predict child oral healthrelated quality of life in adulthood. Health Qual Life Outcomes 2011;9(1):50. DOI: 10.1186/1477-7525-9-50.

5. Giglio JA. Oral health care for the pregnant patient. JCDA 2009;75(1):43-48.

6. Gambhir RS, Nirola A, Gupta T, et al. Oral health knowledge and awareness among pregnant women in India: a systematic review. J Indian Soc Periodontol 2015;19(6):612-617. DOI: 10.4103/0972$124 X .162196$.

7. Mahmoud N, Kowash M, Hussein I, et al. Oral health knowledge, attitude, and practices of Sharjah mothers of preschool children, United Arab Emirates. J Int Soc Prevent Communit Dent 2017;7(6):308314. DOI: 10.4103/jispcd.JISPCD_310_17.

8. Alkhtib A. Knowledge, attitudes, and practices of mothers of preschool children about oral health in Qatar: a cross-sectional survey. Dent J 2018;6(4):51. DOI: 10.3390/dj6040051.

9. Gurunathan D, Moses J, Arunachalam SK. Knowledge, attitude, and practice of mothers regarding oral hygiene of primary school children in Chennai, Tamil Nadu, India. Int J Clin Pediatr Dent 2018;11(4):338343. DOI: 10.5005/jp-journals-10005-1535.

10. Pawar P, Kashyap N. Knowledge, attitude, and practices of mothers related to their oral health status of 6-12 years old children in Bhilai city, Chhattisgarh, India. Eur Sci J 2018;14(21):248-260.

11. Thomas A, Jacob A, Kunhambu D, et al. Evaluation of the knowledge and attitude of expectant mothers about infant oral health and their oral hygiene practices. J Int Soc Prevent Communit Dent 2015;5(5):400-405. DOI: 10.4103/2231-0762.163405.

12. Kierce EA. Adverse pregnancy outcomes and maternal periodontal health. Int J Dent Oral Heal 2017;3(7):69-73. DOI: 10.25141/2471-657X2017-7.0060.

13. Han YW. Oral health and adverse pregnancy outcomes - what's next. J Dent Res 2011;90(3):289-293. DOI: 10.1177/0022034510381905.

14. Chacko V. Self-reported awareness of oral health and infant oral health among pregnant women in Mangalore, India- a prenatal survey. Int J Health Rehabilitat Sci 2013;2(2):109-115.

15. Pregnancy dental concerns. American Dental Association.

16. Nazir MA. Prevalence of periodontal disease, its association with systemic diseases and prevention. Int J Health Sci 2017;1(2):72-80.

17. American Academy of Periodontology statement regarding periodontal management of pregnant patient. American Academy of Periodontology. 2004. 
18. Kurien S. Management of pregnant patient in dentistry. J Int Oral Health 2013;5(1):88-97.

19. ADA policies related to pregnancy.

20. Oral health care for the pregnant adolescent. Am Acad Pediat Dentis 2016;40(6):241-248.

21. Kellesarian SV. Association between prenatal maternal cigarette smoking and early childhood caries. A systematic review. J Clin Exp Dent 2017;9(9):1141-1146. DOI: 10.4317/jced.54064.

22. Hug TE. Clinical and electroretinographic findings in fetal alcohol syndrome. J AAPOS 2000;4(4):200-204. DOI: 10.1067/ mpa.2000.105278.

23. Kuczkowski KM. The effects of drug abuse on pregnancy. Curr Opin Obstet Gynecol 2007;19(6):578-585. DOI: 10.1097/ GCO.0b013e3282f1bf17.

24. Pinto GS. Are maternal factors predictors for early childhood caries? Results from a cohort in Southern Brazil. Braz Dent J 2017;28(3):391397. DOI: 10.1590/0103-6440201601047.

25. Christensen LB, Jeppe JD, Petersen PE. Self-reported gingival conditions and self-care in the oral health of danish women during pregnancy. J Clin Periodontol 2003;30(11):949-953. DOI: 10.1034/j.1600-051X.2003.00404.x.

26. Hullah E. Self-reported oral hygiene habits, dental attendance and attitudes to dentistry during pregnancy in a sample of immigrant women in North London. Arch Gynecol Obstet 2008;277(5):405-409. DOI: 10.1007/s00404-007-0480-8.

27. Assery MK. A 22 year comparison survey of dental knowledge in Al-Jubail antenatal clinic population. Saudi Dent J 2016;28(2):86-90. DOI: 10.1016/j.sdentj.2016.01.002.

28. Ganesh A, Ingle NA, Chaly PE, et al. A survey on dental knowledge and gingival health of pregnant women attending government maternity hospital, Chennai. J Oral Health Comm Dent 2011;5(1):24-30. DOI: 10.5005/johcd-5-1-24.

29. Poureslami HR. Early childhood caries (ECC) an infectious transmissible oral disease. Indian J Pediatr 2009;76(2):191-194. DOI: 10.1007/s12098-008-0216-1.

30. Bastos VA. Mother-to-child transmission of streptococcus mutans: a systematic review and meta-analysis. J Dent 2015;43(2):181-191.

31. Srinivas SK. Periodontal disease and pregnancy outcomes:time to move on. J Womens Health 2012;21(2):121-125.

32. Madianos PN, Bobetsis YA, Offenbacher S. Adverse pregnancy outcomes (APOs) and periodontal disease: pathogenic mechanisms. J Clin Periodontol 2013;40(Suppl. 14):S170-S180. DOI: 10.1902/ jop.2013.1340015.

33. Saini R. Periodontitis: a risk for delivery of premature labor and lowbirth-weight infants. J Nat Sci, Biol Med 2010;1(1):40-42.

34. Nirunsittirat A. Adverse birth outcomes and childhood caries: a cohort study. Commun Dent Oral Epidemiol 2016;44(3):239-247. DOI: 10.1111/ cdoe.12211.

35. Vello MA. Prenatal and neonatal risk factors for the development of enamel defects in low birth weight children. Oral Dis 2010;16(3):257262. DOI: 10.1111/j.1601-0825.2009.01629.x.

36. Sufia S. Dental caries experience in preschool children: is it related to a child's place of residence and family income. Oral Health Prev Dent 2011;9(4):375-379.

37. Mascarenhas AK. Oral hygiene as a risk indicator of enamel and dentin caries. Commun Dent Oral Epidemiol 1998;26(5):331-339. DOI: 10.1111/j.1600-0528.1998.tb01969.x.

38. Elena B, Petr L. Oral health and children attitudes among mothers and school teachers in Belarus. Stomatolog, Balt Dent Maxillofac J 2004;6:40-43. 